\title{
Immunological function of familial Mediterranean fever disease protein Pyrin
}

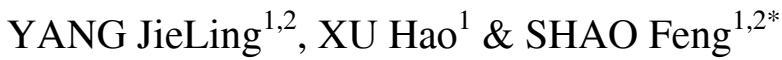 \\ ${ }^{1}$ National Institute of Biological Sciences, Beijing 102206, China; \\ ${ }^{2}$ National Laboratory of Biomacromolecules, Institute of Biophysics, Chinese Academy of Sciences, Beijing 100101, China
}

Received August 6, 2014; accepted September 11, 2014; published online October 10, 2014

\begin{abstract}
Pyrin, encoded by $M E F V$ gene, is conserved in humans and mice. Mutations in the $M E F V$ gene are associated with the human autoinflammatory disease familial Mediterranean fever (FMF). Pyrin can interact with the inflammasome adaptor ASC and induce inflammatory caspase-1 activation in monocytic cells, but the physiological function of Pyrin has been unknown for many years. Here we summarize previous studies of Pyrin function under the context of FMF and immunity, and discuss a recent study demonstrating that Pyrin forms an inflammasome complex for caspase- 1 activation in innate immunity. Pyrin inflammasome detects inactivating modifications of host Rho GTPases by diverse bacterial toxins and infections, including Clostridium difficile glucosylating cytotoxin TcdB, FIC-domain adenylyltransferase effectors from Vibrio parahaemolyticus and Histophilus somni, ADP-ribosylating Clostridium botulinum C3 toxin as well as Burkholderia cenocepacia infection. The mode of Pyrin action, i.e., sensing pathogen virulence activity rather than directly recognizing a microbial molecule, represents a new paradigm in innate immunity.
\end{abstract}

Pyrin, MEFV, Rho GTPase, inflammasome, Guard Model

Citation: $\quad$ Yang JL, Xu H, Shao F. Immunological function of familial Mediterranean fever disease protein Pyrin. Sci China Life Sci, 2014, 57: 1156-1161, doi: 10.1007/s11427-014-4758-3

\section{Pattern recognition in innate immunity}

Both mammals and plants rely on a group of pattern recognition receptors (PRRs) to detect the conserved pathogenassociated molecular pattern (PAMP) or danger-associated molecular pattern (DAMP), and mount an innate immune response to ensure a healthy organism [1,2]. In mammals, the dogma or prevailing concept in innate immune detection is that PRRs directly recognize a microbial product upon infection. This idea is best exemplified by identification and characterizations of the membrane-bound Toll-like receptors (TLRs). TLRs are directly ligated by various PAMPs such as double-stranded RNA (dsRNA) (or Poly IC), components of bacterial cell wall, and bacterial flagellin, which

*Corresponding author (email: shaofeng@nibs.ac.cn) stimulates the transcription of many cytokines, chemokines and inflammatory mediators [3]. It has also been well established that a family of receptor kinases in plants functions similarly as the TLRs in innate immune recognition of microbial products [4]. In the mammalian system, another family of PRRs, most of which are classified as NOD-like receptors (NLR), are proposed to function as cytosolic receptors for various PAMPs or DAMPs $[5,6]$. Human genome encodes more than 20 NLRs that are usually conserved in mice. NLRs generally share the conserved structural organization, consisting of carboxyl-terminal leucinerich repeat domains (LRR), a central nucleotide-binding oligomerization (NOD or NACHT) domain, and a variable amino-terminal protein-protein interaction module, which is a caspase recruitment and activation domain (CARD), a PYRIN domain, or baculovirus inhibitor of apoptosis repeat 
domains (BIR).

Several NLR-family members have been shown to assemble an inflammasome complex; the inflammasome often involves a PYRIN-CARD adaptor protein ASC and activates an inflammatory caspase called caspase-1 [7-9]. Active caspase- 1 further processes interleukin (IL)-1 $\beta / \mathrm{IL}-18$ and induces macrophage inflammatory necrotic death called pyroptosis. Accumulating evidences in recent years suggest that the canonical caspase-1 inflammasome pathway in macrophage and dendritic cells is critical for cytosolic defense against diverse bacterial infections [10,11]. Notably, among all the NLRs, only the BIR-domain containing NAIP subfamily has been demonstrated to serve as the receptor and mediate the assembly of an inflammasome complex $[12,13]$. NAIPs directly recognize cytosolic flagellin and components of bacterial type III secretion system, and activate caspase-1 through the CARD-domain NLR protein NLRC [14-17].

The plant system, in addition to receptor kinase-mediated direct recognition of pathogen-derived molecules, appears to rely more on the intracellular disease resistance $(R)$ protein to sense microbial infection. The $R$ protein shares the similar structure as mammalian NLRs. It has been well accepted that the $R$ protein often acts in an indirect means by detecting pathogen-induced modification of a host protein, i.e., the virulence activity of the pathogen, a model known as "guard hypothesis" [18]. For instance, Pseudomonas syringae type III effectors AvrB and AvrRpm1 can induce modifications of Arabidopsis RIN4, and thereby activate the RPM1 disease resistance protein; AvrRpt2 and AvrPphB proteolytically cleave RIN4 and PBS1, which is detected by RPS2 and RPS5, respectively [18-20]. This "Guard Model" is effective in at least two aspects. First, it can help the host to distinguish the pathogenic from the non-pathogenic bacteria. Second, as different pathogens and toxins often target a few hot-spot pathways or target proteins in the host, perception of the virulence activity rather than the virulence factor itself is advantageous for using a limited number of PRRs to cope with the large number of pathogenic organisms. However, it has not been clear from the literature whether such indirect detection of pathogen virulence activity by PRRs also operates in mammalian innate immunity.

\section{Pyrin and familial Mediterranean fever}

In addition to the canonical NLR family, two cytosolic PYRIN-domain containing atypical NLR proteins, AIM2 and Pyrin, can also mediate inflammasome assembly for caspase-1 activation. AIM2 recognizes cytosolic double-strand DNA (dsDNA) and forms a complex with ASC to activate caspase-1 [21-25]. Pyrin, also called marenostrin and TRIM20, is encoded by the $M E F V$ gene that is highly conserved in humans and mice. Mutations in $M E F V$ cause a hereditary autoinflammatory disease known as familial
Mediterranean fever (FMF). FMF is an autosomal recessive disorder found mostly in ethnic groups living around the Mediterranean basin. The gene responsible for FMF is mapped to $M E F V$ in the short arm of chromosome 16 $[26,27]$ and more than 60 FMF-associated mutations in $M E F V$ have been identified ((http://fmf.igh.cnrs.fr/ISSAID/ infevers/). FMF is clinically characterized by acute peritoneal inflammation, skin rash, pleural and arthritic attack. $M E F V$-encoded Pyrin protein is highly expressed in peripheral blood leukocytes and bone marrow leukocytes, but not other tissues according to the Northern blotting analyses $[27,28]$. Stimulation of monocytes with proinflammatory agents such as interferon- $\gamma$, tumor necrosis factor- $\alpha$ (TNF- $\alpha$ ), and bacterial lipopolysaccharide (LPS) can robustly induce the expression of $M E F V$, suggesting a role in the inflammatory signal cascades [28].

The apparent recessive nature of most $M E F V$ mutations in FMF patients led to an early postulation that the disease mutations are loss-of-function in nature. As a result, it has long been believed that Pyrin is a negative regulatory of a certain inflammatory pathway, and that loss of Pyrin function is responsible for the increased inflammation seen in the FMF patients. This idea results in many controversies regarding the exact function of Pyrin. In particular, genetic deletion of Mefv in mice does not recapitulate the disease symptom; the knockout mice indeed show no obvious phenotypes in the absence of a stimulus and their cytokine and chemokine levels are comparable to those in the wild-type mice $[29,30]$. An initial clue to this puzzle comes from the identification of ASC as a direct binding partner of Pyrin; Pyrin and ASC interact with each other through their N-terminal PYRIN domains [29,31]. Subsequent studies showed that Pyrin overexpression leads to caspase-1 activation in an ASC-dependent manner [32,33]. In addition, cell based reconstitution studies in 293T and THP1 macrophages showed that the disease associated PSTPIP1 mutant proteins can bind to and activate the Pyrin inflammasome [34]. These analyses suggest a possible function of Pyrin in forming an ASC-containing inflammasome complex in innate immunity, but are inconsistent with the idea of Pyrin negatively regulating inflammation and therefore not well accepted at that time. The conflict is resolved by a recent study of generating genetically engineered mice that bear the FMF-causing mutation in mouse Mefv [35]. The knockin mice develop the disease-like symptom with excessive IL-1 $\beta$ release, which is dependent on the presence of an intact Asc allele. These suggest that FMF disease mutations are actually gain-of-function and Pyrin functions together with ASC in an unknown inflammasome pathway.

Pyrin contains a PYRIN domain at the $\mathrm{N}$ terminus (residues 1-100). There has been only one FMF mutation (E148Q) found at the N-terminal region of Pyrin. This mutation, outside of the PYRIN domain, is unlikely to affect ASC binding but may interfere with Pyrin activation by the 
upstream signal. Besides the PYRIN domain, human Pyrin protein possesses a central "pseudo" tripartite motif and a C-terminal B30.2 domain. The tripartite motif in the large TRIM family of ubiquitin ligase proteins is composed of a RING finger followed by one or two B-box zinc fingers and a coil-coil domain. The RING domain in Pyrin lacks the complete set of zinc-binding cysteine and histidine residues and therefore is probably inactive as a ubiquitin ligase. The C-terminal B30.2 domain is functionally important given that two thirds of the known FMF-causing mutations are found in this region [36]. Knock-in mice expressing B30.2 domain mutant Pyrin exhibit serious inflammatory responses and constitutive caspase-1 activation [35]. Thus, the B30.2 domain likely maintains Pyrin in an inactive state in the absence of the stimulus. Structural studies show that the B30.2 domain of Pyrin adopts a $\beta$-sandwich structure composed of two $\beta$-sheet layers [37]. The FMF-associated B30.2 domain mutations are all mapped to the loop regions linking the two $\beta$-sheet layers, consistent with the notion that the B30.2 domain is important for the inactive conformation of Pyrin. Interestingly, the C-terminus of mouse Pyrin lacks the B30.2 domain but bears another domain not recognized by any bioinformatics tools. Given the highly conserved function between human and mouse Pyrin, the C-terminal domain in mouse Pyrin likely fulfils a similar function as the B30.2 domain in human Pyrin.

\section{Pyrin functions as an inflammasome sensor for pathogen modification and inactivation of Rho GTPases}

Despite more than 15 years of research, the physiological function of Pyrin in immunity remained elusive until a recent study from our group [38]. The discovery starts from TcdB, a secreted protein toxin from Clostridium difficile. $C$. difficile is a Gram-positive bacterium responsible for the majority of hospital-acquired infectious diarrhea and antibiotic-associated pseudomembranous colitis. C. difficile causes severe intestinal inflammation when overgrowing in the digestive tract. TcdB, together with the highly similar TcdA toxin, are the two major virulence factor of $C$. difficile $[39,40]$. TcdA and TcdB belong to the large clostridial cytotoxin (LCT) family of $\sim 250-308 \mathrm{kD}$ in size, including the lethal toxin from Clostridium sordellii (TcsL) [41,42]. All proteins in the LCT family are composed of an N-terminal catalytic domain and $\mathrm{C}$-terminal domains responsible for entry and translocation of the toxin into mammalian host cells through the endocytosis pathway. The catalytic domain of TcdB is a glycosyltransferase that modifies and inactivates Rho family of small GTPases in the host, leading to disruption of the actin cytoskeleton structure. Both TcdB and TcsL glucosylate the same threonine residue in the switch I region in Rho GTPases; TcdB can work on all three
Rho subfamilies including Rho, Rac and Cdc42 while TcsL modifies Rac/Cdc42 and some Ras-related GTPases, but not the Rho subfamily [41].

In this new study, we first discover that TcdB but not TcsL can induce robust activation of the caspase- 1 inflammasome in mouse bone marrow macrophages in its Rho-glucosylation activity-dependent manner [38]. This indicates that glucosylation of the Rho subfamily (RhoA/B/ C) may activate an unknown inflammasome pathway. Through a well-designed screen in the 293T cells stably expressing the red fluorescent protein (RFP)-tagged ASC, we identify Pyrin, among a panel of NLR proteins examined, as the specific immune sensor that can support TcdBinduced RFP-ASC foci formation. The function of Pyrin in sensing the Rho glucosylation activity of TcdB and inducing ASC aggregation-dependent caspase- 1 activation is also confirmed in several mouse macrophages/dendritic cells as well as human monocytes. We further generate the Mefv-deficient mice by employing the CRISPR/Cas9mediated genetic targeting and demonstrate that $M e f v^{--}$ macrophage, in contrast to wild-type mouse bone marrow macrophage, is completely resistant to TcdB-induced caspase-1 inflammasome activation.

TcdB and TcsL modification of Rho GTPase leads to disruption of actin cytoskeleton structure. However, we show that cytochalasin D and the actin crosslinking domain (ACD) from Vibrio cholerae RTX toxin [43], two established actin polymerization-inhibiting agents, cannot induce the Pyrin inflammasome activation in mouse bone marrow macrophages. These, together with the inability of TcsL in inflammasome activation, suggest that covalent modification of Rho among the Rho family is likely the cause of TcdB-induced Pyrin activation. We then take advantage of the unique activity of other well-known Rho-modifying effectors or toxins to confirm this hypothesis. Vibrio parahaemolyticus VopS and Histophilus somni IbpA are recently identified effectors that contain catalytic FIC domains and modify a switch I side-chain hydroxyl group in Rho GTPases by a modification called adenylylation or AMPylation [44-46]. VopS adenylylates the same threonine in Rho as TcdB, and the two FIC domains in IbpA modify a nearby tyrosine. C. botulinum $\mathrm{C} 3$ toxin is the first and most established Rho-modifying toxin and catalyzes ADPribosylation of RhoA on Asn-41 that is located at the boarder of switch I region [47-49]. Using both primary bone marrow macrophages and dendritic cell reconstitution systems, we show that VopS, IbpA and C3 toxin all can stimulate Pyrin inflammasome activation, which, similar to TcdB, also require their Rho-modifying activities. These analyses provide a strong support that the Pyrin inflammasome responds to pathogen-induced covalent modification of the switch I region in Rho GTPases and the resulting Rho inactivation in host cells. We further observe that the Rho-inactivation domain (RID) of Vibrio RTX toxin [50], which does not modify Rho but can induce the conversion 
of Rho-GTP to Rho-GDP, appears to harbor no activity of stimulating Pyrin inflammasome. This suggests that Pyrin does not respond to Rho inactivation in general, which is beneficial in avoiding the unwanted deleterious autoinflammation, as Rho GTPases are constantly undergoing conversion between the GTP and GDP-bound forms in the physiological context. The fact that $\mathrm{C} 3$ toxin is highly specific for the Rho subfamily further indicates that modification of the Rho subfamily is the major cause for Pyrin inflammasome activation observed with $\mathrm{TcdB}$ and other Rho-modifying toxins. Our analyses for the first time reveal the normal biological function of the FMF disease protein Pyrin and establish a novel inflammasome pathway in innate immunity.

To further extend the above finding and also probe the physiological significance of Pyrin sensing of Rho modification and inactivation, we examine the role of Pyrin inflammasome in Burkholderia cenocepacia infection. It is known from previous studies that $B$. cenocepacia causes Rho inactivation in its type VI secretion system-dependent manner [51,52]. We confirm this observation and further notice that $B$. cenocepacia infection can induce covalent modification of endogenous RhoA in macrophages. Consistent with that $B$. cenocepacia-induced modification can block further ADP-ribosylation by $\mathrm{C} 3$ toxin, Asn-41 in RhoA in dendritic cells is found to be deamidated into aspartic acid by $B$. cenocepacia in the type VI secretion system-dependent manner. Importantly, we observe that $B$. cenocepacia infection also robustly stimulates inflammasome activation in bone marrow macrophages, which requires Pyrin in the host and the type VI secretion system in the bacteria. When endogenous RhoA, B and C are all efficiently knocked down by small interference RNAs (siRNAs), transient overexpression of the deamidated RhoA (N41D), but not the wild-type version, can evidently drive RFP-ASC foci formation, demonstrating that modification of RhoA is sufficient to induce Pyrin inflammasome activation. Lastly, we find that $B$. cenocepacia replication in $\mathrm{Mefv}^{-1-}$ and $\mathrm{Asc}^{-/-}$macrophages are significantly higher than that in wild-type cells, highlighting a role of Pyrin inflammasome activation in limiting intracellular growth of the bacteria. At the animal level, B. cenocepacia infection of wild-type mice triggers severe lung inflammation, as indicated by inflammatory cell infiltration, appearance of intra-alveolar leukocytes and disruption of normal lung architecture, and importantly these inflammatory responses are strongly attenuated in the infected lungs of $\mathrm{Mefv}^{-1-}$ and $\mathrm{Asc}^{-l-}$ mice. These results establish an important role of the Pyrin inflammasome in innate immune defense against $B$. cenocepacia infection.

\section{Conclusion remarks and perspectives}

Our study establishes the physiological function of the FMF disease gene product Pyrin in innate immune sensing of pathogen modification and inactivation of host Rho GTPases (Figure 1). The modification is diverse in the chemical form, including glucosylation, AMPylation, ADP-ribosylation and deamidation, which takes place on different residues within or around the GTPase switch I region (Figure 1). Consistently, Pyrin does not appear to interact directly with modified Rho [38], suggesting an indirect activation by the virulence activity of bacterial pathogens. This distinguishes Pyrin as an immune sensor from most mammalian PRRs that often directly recognize a microbial product. As mentioned earlier, the $R$ protein in plant innate immunity is established in detecting pathogeninduced modification of a host protein through an indirect mechanism. In this regard, Pyrin appears to guard host Rho GTPases in pathogen-induced modification and inactivation, and the mechanism of Pyrin action somewhat mimics the "Guard Model" in plant innate immunity. Such functional mechanism of Pyrin is particularly advantageous for another important aspect of mammalian innate immune detection. Mammals co-evolve with a large number of microbial organisms which include both pathogenic and non-pathogenic bacteria. Non-pathogenic bacteria such as commensals are sometimes beneficial to or an integrated part of a human body, particularly in an immune-balanced and healthy individual. Specific sensing of pathogen-induced modification of Rho GTPases by Pyrin, together with the direct recognition of the type III secretion apparatus components by the NAIP family of inflammasome receptors $[13,14]$, helps to distinguish pathogenic bacteria from non-pathogenic ones, thereby resolving a long-standing puzzle in innate immune research.

The absence of a direct interaction between Pyrin and modified Rho also suggests that Pyrin senses a signaling event downstream of Rho modification. The signaling mechanism underlying how Rho modification induces Pyrin activation will certainly be the major focus of future studies

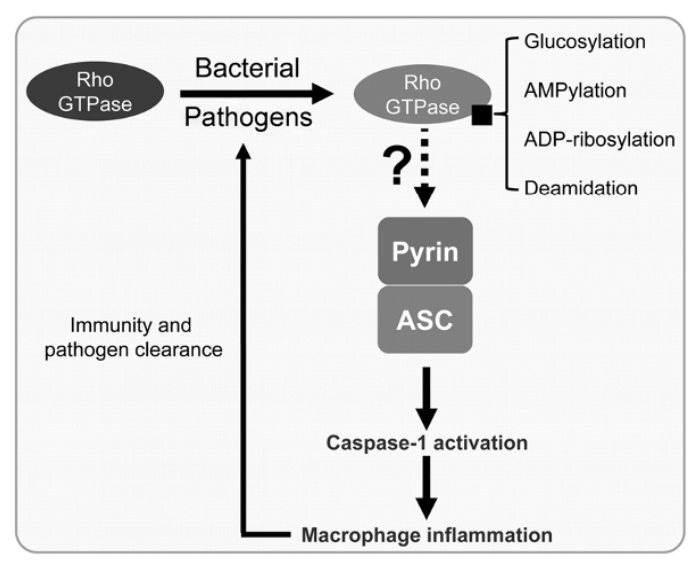

Figure 1 A schematic model for Pyrin inflammasome activation by diverse modifications of Rho GTPases by various pathogen and pathogenic factors. 
on this topic. It is worth noting here that Pyrin has been shown to interact directly with actin and the Pyrin-ASC complex has been shown localized to the actin filamentous structure in previous studies [53,54]. Another study has also identified PSTPIP1 as a binding partner for Pyrin in a yeast two-hybrid screen [55]. Interestingly, PSTPIP1 is proline serine threonine phosphatase-interacting protein involved in actin cytoskeleton organization. Genetic mutations of PSTPIP1 can cause the syndrome of pyogenic arthritis, pyoderma gangrenosum, and acne (PAPA), a dominantly inherited autoinflammatory disorder [56]. Two PAPA syndrome mutations A230T and E250Q in PSTPIP1 can increase the binding to Pyrin. The binding of these mutants to Pyrin can lead to activation of the pyrin inflammasome in a cell-based reconstitution system [34]. Meanwhile, the "pseudo" tripartite motif shown to be responsible for PSTPIP1 binding is also enriched in FMF-associated mutations. These data suggest a possibility that PSTPIP1 might be involved in Pyrin activation by Rho-modifying toxins or effectors, which likely occurs on an actin cytoskeleton platform. Moreover, it is worth mentioning that some bacterial and fungal toxins that induce ribotoxic stress have also been shown recently to activate the human Pyrin inflammasome in a $293 \mathrm{~T}$ reconstitution system [57]. It will be interesting to find out how the ribotoxic stress and the Rho GTPase inactivation pathways might converge to activate the Pyrin inflammasome.

Work in the authors' laboratory was supported in part by an International Early Career Scientist grant from the Howard Hughes Medical Institute and the Beijing Scholar Program to Shao Feng. Other financial supports include the National Basic Research Program of China (2012CB518700), the Strategic Priority Research Program of the Chinese Academy of Sciences (XDB08020202) and National Science Fund for Distinguished Young Scholars (31225002) to Shao Feng.

1 Janeway CA Jr., Medzhitov R. Innate immune recognition. Annu Rev Immunol, 2002, 20: 197-216

2 Takeuchi O, Akira S. Pattern recognition receptors and inflammation. Cell, 2010, 140: 805-820

3 Song DH, Lee JO. Sensing of microbial molecular patterns by Toll-like receptors. Immunol Rev, 2012, 250: 216-229

4 Schwessinger B, Ronald PC. Plant innate immunity: perception of conserved microbial signatures. Annu Rev Plant Biol, 2012, 63: 451-482

5 Chen G, Shaw MH, Kim YG, Nuñez G. NOD-like receptors: role in innate immunity and inflammatory disease. Annu Rev Pathol, 2009, 4 365-398

6 Davis BK, Wen H, Ting JP. The inflammasome NLRs in immunity, inflammation, and associated diseases. Annu Rev Immunol, 2011, 29: 707-735

7 Lamkanfi M, Dixit VM. Inflammasomes and their roles in health and disease. Annu Rev Cell Dev Biol, 2012, 28: 137-161

8 Rathinam VA, Vanaja SK, Fitzgerald KA. Regulation of inflammasome signaling. Nat Immunol, 2012, 13: 333-332

9 Strowig T, Henao-Mejia J, Elinav E, Flavell R. Inflammasomes in health and disease. Nature, 2012, 481: 278-286

$10 \mathrm{Ng}$ TM, Kortmann J, Monack DM. Policing the cytosolbacterial-sensing inflammasome receptors and pathways. Curr Opin
Immunol, 2013, 25: 34-39

11 Vladimer GI, Marty-Roix R, Ghosh S, Weng D, Lien E. Inflammasomes and host defenses against bacterial infections. Curr Opin Microbiol, 2013, 16: 23-31

12 Kofoed EM, Vance RE. Innate immune recognition of bacterial ligands by NAIPs determines inflammasome specificity. Nature, 2011, 477: 592-595

13 Zhao Y, Yang J, Shi J, Gong YN, Lu Q, Xu H, Liu L, Shao F. The NLRC4 inflammasome receptors for bacterial flagellin and type III secretion apparatus. Nature, 2011, 477: 596-600

14 Yang J, Zhao Y, Shi J, Shao F. Human NAIP and mouse NAIP1 recognize bacterial type III secretion needle protein for inflammasome activation. Proc Natl Acad Sci USA, 2013, 110: 14408-14413

15 Rayamajhi M, Zak DE, Chavarria-Smith J, Vance RE, Miao EA. Cutting edge: mouse NAIP1 detects the type III secretion system needle protein. J Immunol, 2013, 191: 3986-3989

16 Tenthorey JL, Kofoed EM, Daugherty MD, Malik HS, Vance RE. Molecular basis for specific recognition of bacterial ligands by NAIP/NLRC4 inflammasomes. Mol Cell, 2014, 54: 17-29

17 Gong YN, Shao F. Sensing bacterial infections by NAIP receptors in NLRC4 inflammasome activation. Protein Cell, 2012, 3: 98-105

18 Jones JD, Dangl JL. The plant immune system. Nature, 2006, 444: 323-329

19 Maekawa T, Kufer TA, Schulze-Lefert P. NLR functions in plant and animal immune systems: so far and yet so close. Nat Immunol, 2011, 12: $817-826$

20 Shao F, Golstein C, Ade J, Stoutemyer M, Dixon JE, Innes RW. Cleavage of Arabidopsis PBS1 by a bacterial type III effector. Science, 2003, 301: 1230-1233

21 Bürckstümmer T, Baumann C, Blüml S, Dixit E, Dürnberger G, Jahn H, Planyavsky M, Bilban M, Colinge J, Bennett KL, Superti-Furga G. An orthogonal proteomic-genomic screen identifies AIM2 as a cytoplasmic DNA sensor for the inflammasome. Nat Immunol, 2009, 10: 266-272

22 Fernandes-Alnemri T, Yu JW, Datta P, Wu J, Alnemri ES. AIM2 activates the inflammasome and cell death in response to cytoplasmic DNA. Nature, 2009, 458: 509-513

23 Hornung V, Ablasser A, Charrel-Dennis M, Bauernfeind F, Horvath G, Caffrey DR, Latz E, Fitzgerald KA. AIM2 recognizes cytosolic dsDNA and forms a caspase-1-activating inflammasome with ASC. Nature, 2009, 458: 514-518

24 Roberts TL, Idris A, Dunn JA, Kelly GM, Burnton CM, Hodgson S, Hardy LL, Garceau V, Sweet MJ, Ross IL, Hume DA, Stacey KJ. HIN-200 proteins regulate caspase activation in response to foreign cytoplasmic DNA. Science, 2009, 323: 1057-1060

25 Hornung V, Latz E. Intracellular DNA recognition. Nat Rev Immunol, 2010, 10: 123-130

26 Consortium FF. A candidate gene for familial Mediterranean fever. Nat Genet, 1997, 17: 25-31

27 Consortium TIF. Ancient missense mutations in a new member of the RoRet gene family are likely to cause familial Mediterranean fever. The International FMF Consortium. Cell, 1997, 90: 797-807

28 Centola M, Wood G, Frucht DM, Galon J, Aringer M, Farrell C, Kingma DW, Horwitz ME, Mansfield E, Holland SM, O'Shea JJ, Rosenberg HF, Malech HL, Kastner DL. The gene for familial Mediterranean fever, MEFV, is expressed in early leukocyte development and is regulated in response to inflammatory mediators. Blood, 2000, 95: 3223-3231

29 Chae JJ, Komarow HD, Cheng J, Wood G, Raben N, Liu PP, Kastner DL. Targeted disruption of pyrin, the FMF protein, causes heightened sensitivity to endotoxin and a defect in macrophage apoptosis. Mol Cell, 2003, 11: 591-604

30 Hesker PR, Nguyen M, Kovarova M, Ting JP, Koller BH. Genetic loss of murine pyrin, the Familial Mediterranean Fever protein, increases interleukin-1beta levels. PLoS One, 2012, 7: e51105

31 Richards N, Schaner P, Diaz A, Stuckey J, Shelden E, Wadhwa A, 
Gumucio DL. Interaction between pyrin and the apoptotic speck protein (ASC) modulates ASC-induced apoptosis. J Biol Chem, 2001, 276: 39320-39329

32 Yu JW, Wu J, Zhang Z, Datta P, Ibrahimi I, Taniguchi S, Sagara J, Fernandes-Alnemri T, Alnemri ES. Cryopyrin and pyrin activate caspase-1, but not NF-kappaB, via ASC oligomerization. Cell Death Differ, 2006, 13: 236-249

33 Seshadri S, Duncan MD, Hart JM, Gavrilin MA, Wewers MD. Pyrin levels in human monocytes and monocyte-derived macrophages regulate IL-1beta processing and release. J Immunol, 2007, 179: 1274-1281

34 Yu JW, Fernandes-Alnemri T, Datta P, Wu J, Juliana C, Solorzano L, McCormick M, Zhang Z, Alnemri ES. Pyrin activates the ASC pyroptosome in response to engagement by autoinflammatory PSTPIP1 mutants. Mol Cell, 2007, 28: 214-227

35 Chae JJ, Cho YH, Lee GS, Cheng J, Liu PP, Feigenbaum L, Katz SI, Kastner DL. Gain-of-function Pyrin mutations induce NLRP3 protein-independent interleukin-1beta activation and severe autoinflammation in mice. Immunity, 2011, 34: 755-768

36 Centola M, Aksentijevich I, Kastner DL. The hereditary periodic fever syndromes: molecular analysis of a new family of inflammatory diseases. Hum Mol Genet, 1998, 7: 1581-1588

37 Weinert C, Grütter C, Roschitzki-Voser H, Mittl PR, Grütter MG. The crystal structure of human pyrin b30.2 domain: implications for mutations associated with familial Mediterranean fever. J Mol Biol, 2009, 394: 226-236

38 Xu H, Yang J, Gao W, Li L, Li P, Zhang L, Gong YN, Peng X, Xi JJ, Chen S, Wang F, Shao F. Innate immune sensing of bacterial modifications of Rho GTPases by the Pyrin inflammasome. Nature, 2014, 513: 237-241

39 Kuehne SA, Cartman ST, Heap JT, Kelly ML, Cockayne A, Minton NP. The role of toxin A and toxin B in Clostridium difficile infection. Nature, 2010, 467: 711-713

40 Lyras D, O'Connor JR, Howarth PM, Sambol SP, Carter GP, Phumoonna T, Poon R, Adams V, Vedantam G, Johnson S, Gerding DN, Rood JI. Toxin B is essential for virulence of Clostridium difficile. Nature, 2009, 458: 1176-1179

41 Jank T, Aktories K. Structure and mode of action of clostridial glucosylating toxins: the ABCD model. Trends Microbiol, 2008, 16: 222-229

42 Just I, Selzer J, Wilm M, von Eichel-Streiber C, Mann M, Aktories K. Glucosylation of Rho proteins by Clostridium difficile toxin B. Nature, 1995, 375: 500-503

43 Cordero CL, Kudryashov DS, Reisler E, Satchell KJ. The Actin cross-linking domain of the Vibrio cholerae RTX toxin directly catalyzes the covalent cross-linking of actin. J Biol Chem, 2006, 281: 32366-32374

44 Mattoo S, Durrant E, Chen MJ, Xiao J, Lazar CS, Manning G, Dixon JE, Worby CA. Comparative analysis of Histophilus somni immunoglobulin-binding protein A (IbpA) with other fic domain-containing enzymes reveals differences in substrate and nucleotide specificities. J Biol Chem, 2011, 286: 32834-32842

45 Worby CA, Mattoo S, Kruger RP, Corbeil LB, Koller A, Mendez JC, Zekarias B, Lazar C, Dixon JE. The fic domain: regulation of cell signaling by adenylylation. Mol Cell, 2009, 34: 93-103

46 Yarbrough ML, Li Y, Kinch LN, Grishin NV, Ball HL, Orth K. AMPylation of Rho GTPases by Vibrio VopS disrupts effector binding and downstream signaling. Science, 2009, 323: 269-72

47 Aktories K. Bacterial protein toxins that modify host regulatory GTPases. Nat Rev Microbiol, 2011, 9: 487-498

48 Aktories K, Weller U, Chhatwal GS. Clostridium botulinum type C produces a novel ADP-ribosyltransferase distinct from botulinum C2 toxin. FEBS Lett, 1987, 212: 109-113

49 Rubin EJ, Gill DM, Boquet P, Popoff MR. Functional modification of a 21-kilodalton G protein when ADP-ribosylated by exoenzyme C3 of Clostridium botulinum. Mol Cell Biol, 1988, 8: 418-426

50 Sheahan KL, Satchell KJ. Inactivation of small Rho GTPases by the multifunctional RTX toxin from Vibrio cholerae. Cell Microbiol, 2007, 9: 1324-1335

51 Flannagan RS, Jaumouillé V, Huynh KK, Plumb JD, Downey GP, Valvano MA, Grinstein S. Burkholderia cenocepacia disrupts host cell actin cytoskeleton by inactivating Rac and Cdc42. Cell Microbiol, 2012, 14: 239-254

52 Rosales-Reyes R, Skeldon AM, Aubert DF, Valvano MA. The Type VI secretion system of Burkholderia cenocepacia affects multiple Rho family GTPases disrupting the actin cytoskeleton and the assembly of NADPH oxidase complex in macrophages. Cell Microbiol, 2012, 14: 255-273

53 Mansfield E, Chae JJ, Komarow HD, Brotz TM, Frucht DM, Aksentijevich I, Kastner DL.The familial Mediterranean fever protein, pyrin, associates with microtubules and colocalizes with actin filaments. Blood, 2001, 98: 851-859

54 Waite AL, Schaner P, Hu C, Richards N, Balci-Peynircioglu B, Hong A, Fox M, Gumucio DL. Pyrin and ASC co-localize to cellular sites that are rich in polymerizing actin. Exp Biol Med (Maywood), 2009, 234: $40-52$

55 Shoham NG, Centola M, Mansfield E, Hull KM, Wood G, Wise CA, Kastner DL. Pyrin binds the PSTPIP1/CD2BP1 protein, defining familial Mediterranean fever and PAPA syndrome as disorders in the same pathway. Proc Natl Acad Sci USA, 2003, 100: 13501-13506

56 Wise CA, Gillum JD, Seidman CE, Lindor NM, Veile R, Bashiardes $\mathrm{S}$, Lovett M. Mutations in CD2BP1 disrupt binding to PTP PEST and are responsible for PAPA syndrome, an autoinflammatory disorder. Hum Mol Genet, 2002, 11: 961-969

57 Yu JW, Farias A, Hwang I, Fernandes-Alnemri T, Alnemri ES. Ribotoxic stress through p38 mitogen-activated protein kinase activates in vitro the human pyrin inflammasome. J Biol Chem, 2013, 288: $11378-11383$

Open Access This article is distributed under the terms of the Creative Commons Attribution License which permits any use, distribution, and reproduction in any medium, provided the original author(s) and source are credited. 